\title{
REFLEXIONES SOBRE EL PENSAMIENTO PROSPECTIVO Y ESTRATÉGICO EN EL MARCO ÉTICO DE LOS NEGOCIOS BAJO LA RESPONSABILIDAD SOCIAL CORPORATIVA EN EL CAPITALISMO
}

\author{
Amado de Jesús Marín Barros ${ }^{1}$
}

\section{RESUMEN}

La reflexión relacionada con el pensamiento prospectivo y estratégico en el marco ética de los negocios bajo la responsabilidad social corporativo en el capitalismo, se ha logrado basado en los distintos pensamientos, entre ellos el enfoque clásico, evolucionista, procesualista y la sistémica, que han sido comparado con estudios de casos de algunas organizaciones que muestran interés en la responsabilidad social empresarial - RSE -, y de la cual se destacan los siguientes resultados:

La relación entre las organizaciones empresariales y el entorno están separados cuando la dinámica del emprendimiento, la creación e innovación y construcción de nuevos conocimientos están ligados a los pensamientos, en este sentido se requiere la implementación permanente de las practicas del pensamiento estratégicos, que permitan en las organizaciones dinamizar el accionar, ligado a un sentir ético y moral que garanticen mantener un acercamiento con las comunidades, para brindar apoyo mediante las responsabilidades sociales, basadas en el común acuerdo de asuntos utilitarios que conlleven a soluciones puntuales útiles.

El problema fundamental se centra en la escasa visión de las organizaciones para la comprensión de las diferencias sociales y la búsqueda ética de soluciones que mitiguen las mismas, que fortalezcan los sectores sociales e introduzcan proyectos y programas basados en la producción de bienes y servicios.

En conclusión se requiere mayor actividad entre la sociedad y el sector empresarial u organizacional con el fin de articular lo relacionado con las interacciones que permitan una responsabilidad social acorde a las necesidades reales del entorno.

Palabras clave: Pensamiento, pensamiento estratégico, ética, responsabilidad social, mercados y negocios. 


\section{REFLECTIONS ON THE PROSPECTIVE AND STRATEGIC THINKING IN BUSINESS ETHICAL FRAMEWORK ON CORPORATE SOCIAL RESPONSIBILITY IN CAPITALISM}

\section{Abstract}

The reflection related to prospective and strategic thoughts in the context of business ethics on corporate social responsibility in capitalism, it has been based on the different thoughts, including the classical approach, evolutionary and systemic precesualista, which have been compared to case studies of some organizations that show interest in corporate social responsibility - CSR - and which are the following results:

The relationship between organizations and the environment are separated when the dynamics of entrepreneurship, creativity and innovation and construction of new knowledge are linked to thoughts, in this sense requires ongoing implementation of strategic thinking practices that allow for energize the actions organizations, linked to a moral and ethical sense to ensure maintaining a rapprochement with the communities to provide support through social responsibilities, based on mutual agreement utilitarian affairs that lead to useful point solutions.

The fundamental problem is focused on the limited vision of the organizations to understand the social and ethical search of solutions to mitigate them, to strengthen social sectors and introduce projects and programs based on the production of goods and services.

In conclusion requires more activity between society and the business sector or organizational in order to articulate the interactions related to enabling social responsibility according to the real needs of the environment.

Keywords: Thinking, Strategic Thinking, Ethics, Social Responsibility and Business Markets

\section{Introducción}

En la ciclo vida de las organizaciones, se enmarcan muchas relaciones que permiten ser exitoso en los procesos comerciales sobre todo en el mundo globalizado de hoy al cual enfrentamos; para lograr tener un adecuado fortalecimiento en ellos se deberán establecer algunos criterios relacionados con el pensamiento estratégico, la ética y la responsabilidad social entre otros; es por ello que se presenta este articulo el cual incorpora cada uno de los elementos que al interactuar hacen que la vida comercial y de negocio sea cada vez más relevante y prospera, en este orden de idea se pretende contextuali- zar al lector en forma precisa algunos elementos que tienen que ver con el pensamiento estratégico, la ética y la responsabilidad social en los negocios.

Es recomendable seguir trabajando el tema y profundizar sobre el mismo, pues la reflexión que se presenta, genera los primeros elementos requeridos para la comprensión de la interacción propuesta. De esta manera desarrollando los temas y profundizando sobre los mismos se generarán mayores valores y se enriquecerán los conocimientos, pues usted ya hace parte 
de la reflexión que se está proponiendo, seguro que con sus aportes fortalecerá los conocimientos, mediante aportaciones que servirán a todos los que se involucren en el proceso. Los conceptos acá reflejados tienen algunas referencias marcadas, las cuales se anunciarán en su momento, pero el autor ha querido establecer una marca sobre su pensamiento y el criterio relevante de lo que constituye el negocio, el pensamiento, la ética, y la responsabilidad empresarial corporativa.

¿Que se conoce en relación a las investigaciones encaminadas al pensamiento prospectivo?

El pensamiento prospectivo se centra frecuentemente a los aspectos económicos de las naciones, debido a que busca establecer métodos que proyecten al futuro a las organizaciones empresariales de negocios en el mundo, abarca problemas de conectividad, problemas humanos, problemas sociales tangibles e intangibles a los cuales se les deberá dar solución, mediante investigaciones que han logrado resultados eficaces para la acción, por el seguimiento y estudio de futuro implementado en todo el mundo a partir de los años 40 y 50.

En este sentido el objetivo fundamental de las investigaciones y acciones encaminadas al pensamiento estratégico en el marco ético de los negocios, se centra en la búsqueda de la equidad social y la justicia, en la que las organizaciones logren actuar con responsabilidad social basados en el fortalecimiento del capital humano como núcleo fundamental.

Basado en lo anterior se generarán mayores expectativas de vida en el individuo, debido a que las organizaciones al pensar en el capital humano, tienden a mejorar la calidad de vida de las regiones, de igual manera permitirán excelentes relaciones entre las organizaciones y las comunidades debido a que el esfuerzo de todos se está reflejando en el ser y la imagen corporativa de cada una de las empresas que implementan acciones responsables tienden a crecer.

Desde el pensamiento ético y el pensamiento prospectivo estratégico se plantea la responsabilidad social organizacional o corporativa, desde lo ético, controlado por un pensamiento estratégico que trata de aprender más con el pensamiento del otro, pero ese otro no es más que un individuo al cual se le busca agregar valor para encontrar un pensamiento más relevante y provechoso, no es un pensamiento estratégico puesto al azar y planteado en mezas de trabajos que le garantice a la comunidad ser incluidas en ellas, es un pensamiento entre pensadores, para así decirlo desde el punto de vista del sistema capitalista, en este orden se pretende comparar lo que las corporaciones vienen desarrollando al interior de sus organizaciones contra el criterio critico que he venido imponiendo desde la academia, tratando de interpretar a cada uno de los autores para así comparar y criticar los alcances que se viven en la realidad, demos una mirada a los conceptos básicos relacionados con el pensamiento y el pensamiento estratégico, el capitalismo, la ética en los negocios y la responsabilidad social empresarial.

El Capitalismo: dentro de los aspectos relevantes que buscan mediar entre las comunidades e influenciar en el consumidor es el pensamiento organizacional éticos, prospectivo y estratégico; estos temas que buscan arraigar la responsabilidad social empresarial con las tesis del capitalismo. En este sentido las organizaciones buscan establecer mecanismos para ingresar a los mercados mediantes propuestas que sean útiles para su accionar, sin embargo el capitalismo no está generando resultados útiles a la sociedad por su propia dinámica, es aconsejable revisar lo que manifiesta el MIT Dictionary of Modern Economics, editado por David W. Pearce, que en su contenido sintetiza el capitalismo de la siguiente manera: 
El capitalismo es un sistema político, social y económico en el que grandes empresas y unas pocas personas acaudaladas controlan la propiedad, incluyendo los activos capitales (terrenos, fábricas, dinero, acciones de la bolsa, bonos). El capitalismo se diferencia del sistema económico anterior, el feudalismo, por la compra del trabajo a cambio de un salario, y ya no por la mano de obra directa que se obtenía por concepto de costumbre, tarea u obligación (cercana a la esclavitud) en el feudalismo. Se diferencia del socialismo mayormente por la predominancia de la propiedad privada, en contraste con la propiedad social de los elementos de producción. En el capitalismo el mecanismo de precios se utiliza como supuesta señal que asigna recursos entre usos distintos. Las distintas formas del capitalismo dependen de, entre otros, el grado al cual se utilice el mecanismo de precios, el grado de competitividad de los mercados y el nivel de participación gubernamental en la economía.

La Responsabilidad social: Para iniciar esta intervención relacionada con la responsabilidad social es conveniente dar respuesta al siguiente interrogante. ¿Será que puede el capitalismo estar atento a las necesidades útiles de las comunidades que garantice un cambio social a la gente del contexto?, es un caso en la que una organización no se visualiza, debido a que su función principal es la recuperación de la inversión con la apropiación de mercados, bajo cualquier tipo de estrategia, en muchos casos producto de la competencia imperfecta, monopolios y oligopolios, en la cual la responsabilidad social queda relegada a lo que las organizaciones llaman productividad sostenible.

Antes de analizar la relación del pensamiento, el capitalismo y la responsabilidad social empresarial RSE revisemos teorías relacionadas con la RSE.
Las diversas teorías relacionadas con la responsabilidad social empresarial - RSE - se establecen acorde a los principios de sus autores, y representadas en las siguientes teorías: Teoría Instrumentales, cuyos actores principales son Friedman, M. (1970), Murray, K. y Montanari, J. (1986), Lizt, R. (1996) y Porter, M. y Kramer, M. (2002, 2006); La teoría Integradora cuyos actores son: Carroll, A. (1979), Jones, T. (1980), Vogel, D. (1986) y Wilcox. D. (2005); La teoría de carácter político de Davis, K. (1960), Donaldson,T. y Dunfee, T.W. (1994) y Wood, D. y Lodgson, J. (2002), y por último la teoría sobre la ética y la moral de los negocios con la presencia de Freeman, R.E. (1983), Kaku, R. (1997), UN Global Compact (1999) y Chomali, F. y Majluf, N. (2007).

¿Qué es lo más importante de este tipo de teorías de responsabilidad social?, para ello es importante analizar las características que representa cada una de ellas:

El enfoque sobre la teoría instrumental refiere lo siguiente:

«Estas teorías se enfocan en el estudio de las actividades de RSE que permiten crear una mayor riqueza empresarial, su foco es alcanzar objetivos económicos por sobre las actividades sociales». Friedman (1970), Murray y Montanari (1986), Lizt (1996), y Porter y Kramer (2002).

Adicionalmente tienen la RSE tiene 4 argumentos que lo justifican, primero la Obligación moral, si invertimos y recogemos capital debemos invertir en las regiones; el segundo argumento es la sustentabilidad, relacionada con lo económico, lo social y lo medioambiental; el tercer elemento es la licencia para operar, trabajan sus decisiones basados en los temas de los diversos grupos de interés; y el cuarto argumento es la reputación, para ello se requiere validar su imagen ante las comunidades. 
El enfoque sobre la teoría integradora hace referencia según sus autores Carroll (1979), Jones (1980), Vogel (1986) y Wicox (2005):

Estas teorías explican el desarrollo de actividades de responsabilidad social empresarial - RSE - por el deseo de integrar diversas demandas sociales. Entre estas demandas destaca el cumplimiento de las leyes, las políticas públicas y la gestión balanceada de los intereses particulares de los grupos de interés de una empresa. Carroll (1979), Jones (1980), Vogel (1986) y Wicox (2005).

La relación social de Carroll (1979) consiste en ¿Qué se incluye en RSE? ¿Cuáles son los asuntos sociales que las empresas deben atender? y ¿Cuál es la filosofía organizacional o modo de receptividad social? Su actividad apunta a la unificación de conceptos hacia la responsabilidad social y un modelo de desempeño social.

La teoría de carácter político se expresa según sus autores Davis (1960), Donaldson y Dunfee (1994), y Wood y Logson (2002) está basada en lo siguiente:

En lo referente a las investigaciones asociadas a las teorías de carácter político, es decir, aquellas que prestan atención al poder social que adquieren las empresas en la medida que más influyen en una sociedad, presentaremos tres estudios intentan plasmar la necesidad del uso responsable del poder social en el ámbito político de los negocios, cuyo autores son: Davis (1960), Donaldson y Dunfee (1994), y Wood y Logson (2002).

La teoría sobre la ética y moral en los negocios, es interpretada por Freeman (1983), Kaku (1997), Annan (1999), y Chomalí y Majluf (2007) de la siguiente manera:

La teoría sobre la ética y la moral pretende plasmar las bases del desarrollo de actividades socialmente responsables bajo la mirada de perseguir fines éticos o morales. Esto quiere decir que las consideraciones para realizar actividades de RSE estarán fundamentadas principalmente en parámetros de derechos universales o espirituales, y no en términos estrictamente económicos. Se destacan bajo esta mirada los valores éticos y el espíritu de servicio a la sociedad. Freeman (1983), Kaku (1997), Annan (1999), y Chomalí y Majluf (2007).

A pesar de que existen elementos económicos que requieren ser atendidos ante la inversión realizada, se genera una mirada a los valores éticos y espirituales, en las organizaciones y dentro de los requerimientos para la sociedad, fundamenta la moral, lo ético y lo espiritual como elementos decisivos para el desarrollo social.

Camejpo y Ceja expresan lo siguiente, en la revista Nómada, sobre la responsabilidad social empresarial RSE:

El análisis de la Responsabilidad Social en las organizaciones como categoría de estudio conlleva: En primer lugar a entender que su puesta en práctica conduce a las organizaciones a desarrollar una visión integral de futuro, en la que no sólo está incorporada la comunidad y sus trabajadores sobre la cual se asientan, sino también su país o su sociedad. En segundo lugar, que nazca una nueva forma de organización que promueva el liderazgo internamente, a los fines de que los mismos contribuyan a reforzar la misión que se traza la organización y logren la descentralización de los niveles de autoridad con el fin de perfeccionar los niveles de productividad y competitividad empresarial. Desde este ámbito de estudio es necesario recalcar que en los últimos años se han dado a conocer proyectos de alcance social desde la Organización de las Naciones Unidas (ONU) y desde la Organización Internacional del Trabajo (OIT) lo que 
ha permitido que la Responsabilidad Social se convierta en un punto de referencia sustantivo para el debate acerca del crecimiento y desarrollo de los países. Sus alcances Ilegan al compromiso que debe tener y manifestar las organizaciones en términos de responsabilidad tributarias, de medios de comunicación, de derechos humanos así como de la mejora de la calidad de vida de los propios empleados. La responsabilidad social empresarial es una tendencia de acción social, que a escala mundial estimula la participación solidaria de la empresa privada en el desarrollo sustentable y humano del las comunidades donde presta sus servicios y desarrollan sus actividades.

Ahora ¿como interactuar con responsabilidad social empresarial, con mecanismos éticos de negocio?

Se puede interactuar con pensamiento estratégico generalizado que apunten a la moralidad y al entendimiento entre organizaciones empresariales y contexto. Necesariamente se requiere de mayor sensibilidad empresarial, mayor comprensión de la cultura y el desarrollo de los pueblos y sobre todo mayor interacción con las comunidades, no regalando auxilios, sino profundizando en el mejoramiento de las calidades de vida de las comunidades en las cuales establecen su accionar, la responsabilidad social no puede estar limitada a mitigar los daños ecológicos que se han generado, la responsabilidad social debe ir más allá, fundamentalmente a no seguir deteriorando el medio ambiente, ni la destrucción del hombre como ser social.

Generar mejores condiciones de vida de las comunidades se constituye en elementos indiscutible de la responsabilidad social, en este sentido las organizaciones deben apuntar al pensamiento prospectivo para el fortalecimiento del capital humano, generar hombres con capital intangible proporcionará resultados incalculables para la humanidad y el desarrollo productivo de las regiones, pensar de manera limitada, genera un estancamiento que no favorece a las regiones antes por el contrario atrasa los procesos.

La responsabilidad social empresarial, será la clave en la medida en que sea el capital humano quien de manera directa reciba la influencia de estas acciones mediante el fortalecimiento de recursos intangibles.

\section{Conclusiones}

La responsabilidad social, la ética, el negocio y los recursos están tomados de la mano, tienen que actuar juntos con el fin de lograr los objetivos esperados, el capital humano, es clave para desarrollar acciones que determinen el crecimiento organizacional y no solo basta con apoyar a los miembros de la organización, sino que hay que estar atento a las comunidades con el fin de generar culturas que propendan por inquietudes sobre los nuevos conocimientos y nuevos sistemas que posibiliten la producción y con ella la generación de ingresos para todos de manera abierta; cerrarse es buscar una dinámica monopolista que hace del mercado un sistema imperfecto.

El buen desarrollo organizacional depende de la forma como las organizaciones enfrenta los aspectos sociales internos y del contexto, no importa la estrategia utilizada, lo más importante es determinar las acciones útiles en las cuales los distintos actores se van a ver beneficiados, aun cuando se empleen diversos criterios y formas de actuar, lo importante es generar mayores valores en ellos, en tal sentido hay que revisar de qué manera se están direccionando el pensamiento, debido a que la búsqueda de una adecuada solución a la interacción con los negocios mediante la responsabilidad social está encaminada a resaltar soluciones sociales, que de llegarse a concretar brindarán una proyección significativa a favor de las comunidades y las brechas en el sistema productivo, empresarial y social serán recortadas. 


\section{Referencias}

Álvarez Gálvez, Íñigo. (2010). El utilitarismo y los derechos humanos. Madrid: Plaza y Valdés.

UNESCO, 2013. Declaración de los derechos humanos. disponible en http://portal.unesco.org/es/ev.php-URL ID=26053\&URL DO=DO TOPIC\&URL SECTION=201. html consultado el 21 de marzo de 2013.

Universidad Libre. S.f. Debido proceso, disponible en http://www.unilibrepereira.edu.co/catehortua/posgrados/archivos $2 /$ Trabajo $\% 20$ debido $\% 20$ proceso $\% 20$ curso $\% 202 \% 20$ constitucional $\% 20 \% 20202 \% 20$ dejado\%20agosto\%2006\%20de\%202008.pdf consultado el 22 de marzo de 2013.

Eumed.net. s.f. Fuerza empresarial para la prosperidad de Bogotá y la Región, Disponible en http://www. eumed.net/tesis-doctorales/2010/prc/Cultura\%20 Organizacional.htm consultado el 21 de marzo de 2013 disponible

Castro Heredia, Javier Andrés; Urrea Giraldo, Fernando; Viáfara López, Carlos Augusto. 2009. Un breve acercamiento a las políticas de Acción Afirmativa: orígenes, aplicación y experiencia para grupos étnico-raciales en Colombia y CaliRevista Sociedad y Economía. 16: 159-170. Disponible en: http://www.redalyc.org/pdf/996/99612491009.pdf consultado el 21 de marzo de 2013.

Felaban. S.f. Como se audita la ética, disponible en http://www.felaban.com/pdf/teleconferencias/clain/webconfer/Como\%20se\%20audita.pdf consultado el 25 de marzo de 2013
Junta de Andalucía. S.f. Discriminación como clarificación de conceptos, disponible en http://www.juntadeandalucia. es/averroes/vertie/forum/discrimi.htm\#discriminaciónsocial consultado el 24 de marzo de 2013

Organización Internacional del Trabajo OIT. S.f. Género, Diversidad e Igualdad, disponible en http://www.oit.org/ public/spanish/dialogue/actemp/whatwedo/projects/diversity.htm consultado el 23 de marzo de 2013

Organización Internacional del Trabajo OIT. S.f. Seguridad y Salud en el Trabajo, disponible en http://www.ilo. org/global/topics/safety-and-health-at-work/lang--es/index.htm consultado el 23 de marzo de 2013

Wikipedia, El primer párrafo fue tomado de The MIT Dictionary of Modern Economics, David W. Pearce (editor), The MIT Press, Cambridge, Massachusetts, El Capitalismo, disponible en http://www.ciepac.org/neoliberal/esp/ capitalismo.html consultado el 12 de abril de 2013

Wikipedia, la enciclopedia libre, Deontología, disponible en http://es.wikipedia.org/wiki/Deontolog\%C3\%ADa. Consultado el 21 de marzo de 2013

Wikipedia: Método Científico, disponible en http:// es.wikipedia.org/wiki/M\%C3\%A9todo cient\%C3\%ADfico Consultado el 15 de mayo de 2013

Capitalismoprom12. S.f. Ventajas y Desventajas del Capitalismo, disponible en http://capitalismoprom12.blogspot.com/2012/08/ventajas-y-desventajas-del-capitalismo.html conusltado el 10 de abril de 2013 